\title{
Análisis bibliométrico de la participación estudiantil en publicaciones de artículos científicos en revistas de ciencias de la salud indizadas en SciELO Bolivia, periodo 2010-2016
}

\author{
Bibliometric analysis of student participation in publications of scientific articles in health sciences journals \\ indexed in SciELO Bolivia, period 2010-2016
}

Aarón Eduardo Carvajal Tapia ${ }^{1}$ Eduardo Carvajal Rodríguez ${ }^{2}$

\begin{abstract}
Resumen
Los estudios en relación a la participación estudiantil en revistas indizadas han sido y continúan siendo tema de estudio por su impacto como indicador de producción y esfuerzo científico de una región, considerando que muchos hitos históricos en medicina fueron por estudiantes. Objetivos: determinar a través de un análisis bibliométrico la participación estudiantil en publicaciones de artículos científicos en revistas de ciencias de la salud indizadas en SciELO Bolivia en el periodo comprendido 2010 al 2016. Métodos: estudio bibliométrico, transversal y descriptivo, basado en los registros de publicaciones de revistas de ciencias de la salud de periodicidad semestral indizadas en la base de datos de SciELO Bolivia, incluidos todos los artículos originales, artículos de revisión, casos clínicos, imágenes médicas y cartas al editor durante el periodo de estudio. Resultados: de 471 artículos publicados por las revistas seleccionadas 168 artículos correspondieron a participación estudiantil (35,67\%). La revista con mayor participación de estudiantes se sitúa en la Revista Científica Ciencia Médica con 126 publicaciones (75,00\%), seguida de la Gaceta Médica Boliviana con 34 publicaciones (20,24\%) ambas pertenecientes a la Facultad de Medicina de la Universidad Mayor de San Simón y con menor participación de estudiantes la Revista Médica La Paz con 8 publicaciones (4,76\%). Conclusiones: existe escasa presencia de participación científica estudiantil en Bolivia, a excepción de la Revista Científica Ciencia Médica que da mayor cobertura a los estudiantes con un número importante de publicaciones, seguida de la Gaceta Médica Boliviana.
\end{abstract}

Palabras claves: estudiantes de medicina, investigación universitaria, publicación científica

\section{Abstract}

Studies in relation to student participation in indexed journals have been and continue to be studied because of their impact as an indicator of scientific production and effort in a region, considering that many historical milestones in medicine were made by students. Objective: to determine, through a bibliometric analysis, student participation in publications of scientific articles in health sciences journals indexed in SciELO Bolivia from 2010 to 2016. Methods: bibliometric, cross-sectional and descriptive study, based on the records of publications of health sciences journals of biannual periodicity indexed in the database of SciELO Bolivia, including all original articles, review articles, clinical cases, medical images and letters to the editor during the study period. Results: of 471 articles published by the magazines selected, 168 articles corresponded to student participation (35.67\%). The journal with the highest participation of students was the Revista Científica Ciencia Médica with 126 publications (75.00\%), followed by the Gaceta Médica Boliviana with 34 publications (20.24\%), both belonging to the School of Medicine of the Universidad Mayor of San Simón and with less student participation of the Revista Médica La Paz with 8 publications (4.76\%). Conclusions: : there is a scarce presence of student scientific participation in Bolivia, with the exception of the Revista Científica Ciencia Médica that gives greater coverage to students with a significant number of publications, followed by the Gaceta Médica Boliviana.

Keywords: medical students, university research, scientific publication

L os estudios en relación a la participación estudiantil en revistas indizadas, tales los casos de publicaciones realizadas; en Chile ${ }^{1}$, Colombia ${ }^{2}$, Paraguay ${ }^{3}$, Perú ${ }^{4}$ y Venezuela $^{5}$ ha permitido conocer la importancia de la contribución científica de estudiantes de las ciencias de la salud, a fin de establecer el nivel de aportación y generación de recursos humanos con cultura de investigación al campo del conocimiento científico ${ }^{6}$.

En el caso de las universidades y su vínculo estrecho

${ }^{1}$ Médico cirujano, Facultad de Medicina de la Universidad Mayor de San Andrés - La Paz, Bolivia. Médico residente de pediatría, Hospital de Niños "Dr. Mario Ortiz Suarez"

${ }^{2}$ Estadístico matemático, Universidad Técnica de Oruro, Ministerio de Desarrollo Rural y Tierras (MDRyT). La Paz, Bolivia.

${ }^{\star}$ Correspondencia a:Aarón Eduardo Carvajal Tapia

Correo electrónico: aecarvajal3@gmail.com

Recibido el 09 de noviembre del 2017. Aceptado el 25 de enero del 2018. con la investigación sigue siendo el principal factor para la obtención de indicadores de alta calidad académica ${ }^{7}$, en la que el rol del docente debe sobresalir como investigador, para que sea evaluado a través de sus producciones científicas más que a su rol de docente ${ }^{8}$, considerando que varias Facultades de Medicina en América Latina, reconocen que la enseñanza de la investigación es superflua e incompleta si no se enseña a los estudiantes el proceso de cómo publicar?.

Vale decir, la investigación, conduce a ser parte del conocimiento, hacer crecer una profesión, fortalecer una disciplina, beneficiar a la población y formar sujetos apasionados por profundizar mucho más sus razonamiento, con pensamiento crítico y creatividad, de modo que permita la formación de futuros profesionales apasionados por la búsqueda de respuestas ${ }^{10}$, considerando que, la investigación y posteriormente su publicación, sea manifestada a la 
Tabla 1. Revistas de ciencias de la salud indizadas a SciELO Bolivia

\begin{tabular}{|c|c|c|c|c|}
\hline Revista & $\begin{array}{c}\text { Números } \\
\text { publicados }\end{array}$ & Institución & Periodicidad & Actualizada \\
\hline $\begin{array}{l}\text { Gaceta Médica } \\
\text { Boliviana }\end{array}$ & 14 & $\begin{array}{l}\text { Facultad de Medicina de la Universidad Mayor } \\
\text { de San Simón }\end{array}$ & Semestral & $\mathrm{Si}$ \\
\hline $\begin{array}{c}\text { Revista } \\
\text { Científica } \\
\text { Ciencia Médica }\end{array}$ & 14 & $\begin{array}{l}\text { Sociedad Científica de Estudiantes de Medicina, } \\
\text { Facultad de Medicina de la Universidad Mayor } \\
\text { de San Simón }\end{array}$ & Semestral & $\mathrm{Si}$ \\
\hline $\begin{array}{c}\text { Revista Médica } \\
\text { La Paz }\end{array}$ & 14 & Colegio Médico Departamental de La Paz & Semestral & $\mathrm{Si}$ \\
\hline
\end{tabular}

comunidad social, científica y general, es decir, "lo que no se publica no existe"10-11.

A pesar de innumerables esfuerzos de diferentes estudiantes en Bolivia como congresos y cursos de investigación con diferentes temáticas en cuestión al tema, no es un punto que ha llevado un interés creciente, traducido al número reducido de investigadores y de publicaciones, considerando que son muchas las revistas que no exigen un grado académico como criterio para publicar un trabajo, más si la calidad del mismo.

En este sentido, actualmente en Bolivia existe muy poca o ninguna publicación sobre la participación científica de estudiantes de pregrado como autores de artículos de investigación, en comparación a otros países que fomentan desde hace varios años, la medición constante de publicaciones de estudiantes de pregrado en revistas indizadas, para conocer el grado de contribución, influencia de la universidad en la formación del perfil del egresado y analizar posteriormente si se está cumpliendo con lo esperado.

Para cuyo efecto este trabajo lleva por propósito fortalecer la producción científica en Bolivia, a través de estudios previos que ayuden a identificar diferentes factores que repercuten en la producción científica de Bolivia, enfatizando principalmente la participación científica desde el pregrado como elemento útil de formación académica científica.

Por lo señalado, el presente estudio tiene como objetivo determinar a través de un análisis bibliométricola participación estudiantil en publicaciones de artículos científicos en revistas de ciencias de la salud indizadas en SciELO Bolivia, de manera que permita la identificación, análisis y comparación de las características entre revistas e instituciones que promueven la misma, en el periodo comprendido de 2010 al 2016.

\section{Material y métodos}

El presente estudio se circunscribe a un estudio bibliométrico, transversal y descriptivo, basado en los registros de publicaciones de revistas de ciencias de la salud de periodicidad semestral indizadas en la base de datos de SciELO Bolivia, en las que se incluye todos los números publicados en el periodo comprendido de 2010-2016 de las revistas seleccionadas.

En este sentido, se consideró los siguientes tipos de publicaciones como: artículos originales, artículos de revisión, casos clínicos, imágenes médicas y cartas al editor, excluyendo los artículos que no consignaron la filiación de sus autores.

Para este efecto, se recolectó la información haciendo uso de la página web de la base de datos SciELO Bolivia (http://www.scielo.org), a partir del cual se procedió a la sistematización y clasificación del número de publicaciones del periodo de estudio establecido, el tipo de contribución y la participación estudiantil en cada una de ellas. Posteriormente se creó una base de datos en Microsoft Excel 2010, con la que se realizó la construcción de tablas y figuras de salidas y el análisis descriptivo.

\section{Resultado}

En la revisión de la base de datos de SciELO-Bolivia, en la materia de ciencias de la salud se encontraron cuatro revistas indizadas, de las cuales solo tres cumplen la periodicidad semestral, misma que están actualizadas al periodo de estudio (Tabla 1).

Por otro lado, se efectuó la revisión de 42 números con un total de 471 artículos publicados, de las cuales 225 (47,77\%)

Tabla 2. Número y porcentaje de publicaciones por tipo de revistas de ciencias de la salud según participación de estudiantes y profesionales

\begin{tabular}{|c|c|c|c|c|c|c|c|c|}
\hline \multirow[t]{2}{*}{ Descripción } & \multirow{2}{*}{\multicolumn{2}{|c|}{$\begin{array}{c}\text { Gaceta Médica } \\
\text { Boliviana } \\
\text { NúmeroPorcentaje }\end{array}$}} & \multicolumn{2}{|c|}{$\begin{array}{l}\text { Revista Científica } \\
\text { Ciencia Médica }\end{array}$} & \multicolumn{2}{|c|}{$\begin{array}{c}\text { Revista Médica La } \\
\text { Paz }\end{array}$} & \multirow[t]{2}{*}{ Total } & \multirow[t]{2}{*}{ Total (\%) } \\
\hline & & & Número & Porcentaje & Número & Porcentaje & & \\
\hline A.O. Estudiantes & 15 & 19,48 & 57 & 74,03 & 5 & 6,49 & 77 & 100,00 \\
\hline A.O. Profesionales & 72 & 48,65 & 20 & 13,51 & 56 & 37,84 & 148 & 100,00 \\
\hline Total A.O. & 87 & 38,67 & 77 & 34,22 & 61 & 27,11 & 225 & 100,00 \\
\hline A.R. Estudiantes & 1 & 5,88 & 16 & 94,12 & 0 & 0,00 & 17 & 100,00 \\
\hline A.R. Profesionales & 11 & 28,95 & 5 & 13,16 & 22 & 57,89 & 38 & 100,00 \\
\hline Total A.R. & 12 & 21,82 & 21 & 38,18 & 22 & 40,00 & 55 & 100,00 \\
\hline C.C. Estudiantes & 13 & 30,95 & 26 & 61,90 & 3 & 7,14 & 42 & 100,00 \\
\hline C.C. Profesionales & 46 & 46,94 & 13 & 13,27 & 39 & 39,80 & 98 & 100,00 \\
\hline Total C.C. & 59 & 42,14 & 39 & 27,86 & 42 & 30,00 & 140 & 100,00 \\
\hline Total I.M. & 1 & 5,00 & 19 & 95,00 & 0 & 0,00 & 20 & 100,00 \\
\hline I.M. Estudiantes & 0 & 0,00 & 16 & 100,00 & 0 & 0,00 & 16 & 100,00 \\
\hline I.M. Profesionales & 1 & 25,00 & 3 & 75,00 & 0 & 0,00 & 4 & 100,00 \\
\hline Total C.E. & 17 & 54,84 & 13 & 41,94 & 1 & 3,23 & 31 & 100,00 \\
\hline C.E. Estudiantes & 5 & 31,25 & 11 & 68,75 & 0 & 0,00 & 16 & 100,00 \\
\hline C.E. Profesionales & 12 & 80,00 & 2 & 13,33 & 1 & 6,67 & 15 & 100,00 \\
\hline Total estudiantes & 34 & 20,24 & 126 & 75,00 & 8 & 4,76 & 168 & 100,00 \\
\hline Total profesionales & 142 & 46,86 & 43 & 14,19 & 118 & 38,94 & 303 & 100,00 \\
\hline Total general & 176 & 37,37 & 169 & 35,88 & 126 & 26,75 & 471 & 100,00 \\
\hline
\end{tabular}


Figura 1: Participación porcentual de estudiantes y profesionales según revistas

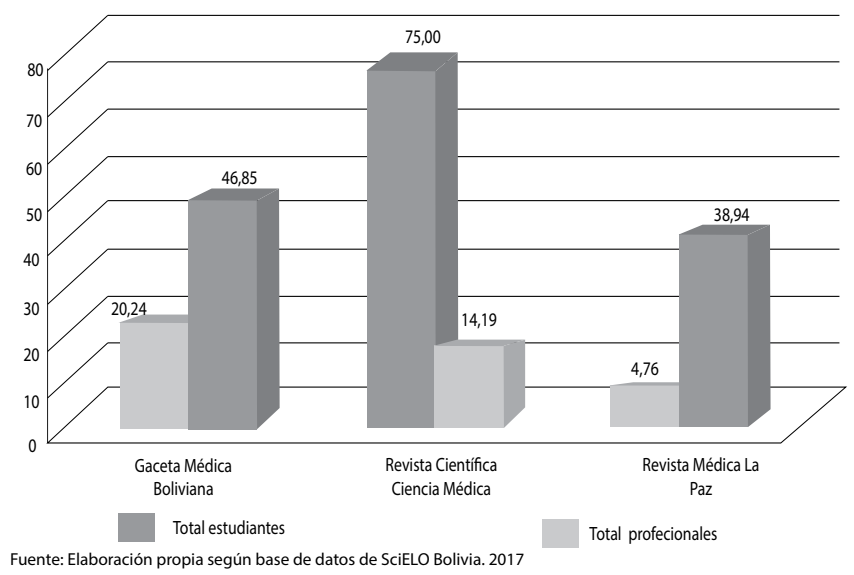

corresponden a Artículos Originales, 55 (11,68\%) Artículos de Revisión, 140 (29,72\%) Casos Clínicos, 20 (4,25\%) Imágenes médicas y $31(6,58 \%)$ a Cartas al editor (Tabla 2).

En lo que corresponde a la contribución por revistas, la Gaceta Médica Boliviana alcanzo un aporte de 176 artículos (37,37\%) en el periodo de 2010 al 2016, seguida de la Revista Científica Ciencia Médica con 169 artículos $(35,88 \%)$ y la Revista Médica La Paz con 126 publicaciones (26,75\%)

En el caso de la Gaceta Médica Boliviana, su participación en relación al total de artículos originales alcanza 38,67\% (87 publicaciones), artículos de revisión 21,82\% (12 publicaciones), casos clínicos 42,14\% (59 publicaciones), imagen médica 5,00\% (1 publicación) y cartas al editor $54,84 \%$ (17 publicaciones). En cambio, la Revista Científica Ciencia Médica cuyo aporte de artículos originales fue de $34,22 \%$ (77 publicaciones), artículos de revisión 38,18\% (17 publicaciones), casos clínicos 27,86\% (39 publicaciones), imágenes médicas $95,00 \%$ (19 publicaciones) y cartas al editor $41,94 \%$ (13 publicaciones) y en el caso de la Revista Médica La
Paz su participación en relación al total general corresponde para los artículos originales 27,11\% (61 publicaciones), artículos de revisión 40,00\% (22 publicaciones), casos clínicos 30,00\% (42 publicaciones), cartas al editor 3,23\% (1 publicación) e imágenes médicas ninguna (Tabla 2).

En cuanto a la presencia de estudiantes de medicina que publicaron según revistas, se observa en el caso de la Gaceta Médica Boliviana, la publicación de 15 artículos originales de un total de 87 artículos, seguida de 12 artículos de revisión de los cuales 1 corresponde a la participación de estudiantes, en los casos clínicos de 59 artículos, 13 corresponden a la participación estudiantil, en imagenes médicas no se observa contribución alguna y de 17 cartas al editor 5 corresponden a la presencia de estudiantes (Tabla 2).

Por su parte, en la Revista Científica Ciencia Médica de un total de 77 artículos originales, 57 trabajos corresponde a la participación estudiantil, entre tanto de 21 artículos de revisión 16 concierne a los estudiantes, en casos clínicos de 39 artículos, 26 se observa la participación estudiantil, de 19 imágenes médicas 16 son publicadas por estudiantes y de 13 cartas al editor 11 corresponden a la participación de estudiantil (Tabla 2).

En el caso de la Revista Médica La Paz de 61 artículos originales, en cinco se observó participación estudiantil, en artículos de revisión, imagen médica y cartas al editor no se evidencia participación estudiantil y en casos clínicos de 42 trabajos, en tres se observa la presencia de estudiantes (Tabla 2).

Con relación a la participación porcentual de estudiantes y profesionales según revistas, se observa en el caso de los estudiantes de pregrado una contribución poco significativa en la Revista de Gaceta Médica Boliviana y Revista Médica La Paz con el 20,24\% y 4,75\%, respectivamente. Sin embargo, la Revista Científica Ciencia Médica presenta una mayor participación de los estudiantes con el 75,00\%, situación que podría deberse a que esta revista esta compuesta por estudiantes y profesionales de las ciencias de la salud, que dan mayor oportunidad a ambos actores (Figura 1).

Figura 2. Participación porcentual de estudiantes y profesionales por tipo de publicaciones según revistas

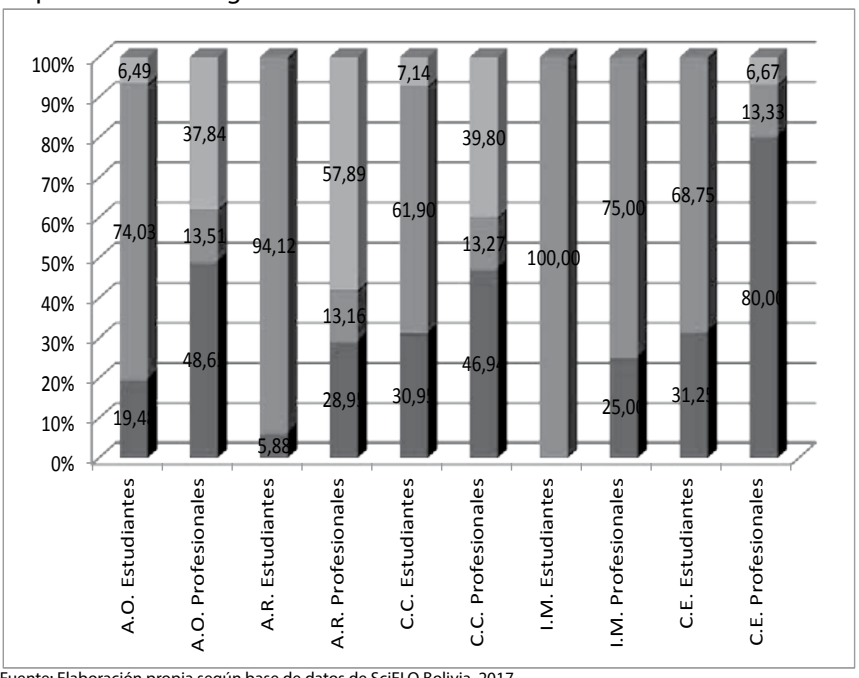


La contribución de publicaciones por parte de los profesionales, sobresalen con una participación del 46,86 \% en la Gaceta Médica Boliviana y 38,94\% en la Revista Médica La Paz, a excepción de la Revista Científica Ciencia Médica que alcanza solo el $14,19 \%$ respecto del total publicaciones (471).

Concerniente a la participación de los estudiantes y profesionales por tipo de publicaciones según revistas, destaca la presencia de estudiantes con el 74,03\% en el caso de Artículos Originales en la Revista Científica Ciencia Médica, en cambio los profesionales presenta una contribución relativamente importante con el $48,65 \%$ en la Gaceta Médica Boliviana y 37,84\% en la Revista Médica La Paz; por su parte, en lo que se refiere a Artículos de Revisión los estudiantes tienen una presencia significativa en la Revista Científica Ciencia Médica con el $94,12 \%$ y los profesionales sobresalen con el 57,89\% en la Revista Médica La Paz. Respecto a la presencia de los estudiantes en las publicaciones de Casos Clínicos muestra una participación del 61,90\% en la Revista Científica Ciencia Médica y en el caso de los profesionales se distribuye su aporte con el 46,94\% en la Gaceta Médica Boliviana y 39,80\% Revista Científica Ciencia Médica (Figura 2).

En cuanto a Imagenes médicas, tanto estudiantes como profesionales sobresalen con una participación significativa del 100,00\% y 75,00\% en la Revista Científica Ciencia Médica; y por último, acerca de las Cartas al Editor la proporción de estudiantes que presentan mayor número de publicación alcanza el 68,75\% en la Revista Científica Ciencia Médica y los profesionales con el 80,00\% en la Gaceta Médica Boliviana (Figura 2).

\section{Discusión}

En este estudio, se evidenció la participación de estudiantes de medicina en publicaciones de tres revistas de ciencias de la salud indizadas a SciELO Bolivia: de un total de 471 publicaciones (Artículos originales, Revisión, Caso clínico, Imagen médica y Carta al editor) 168 presentan a participación estudiantil en el periodo 2010-2016 con participación del $35,67 \%$ del total general de las tres revistas consultadas.

En cuanto al número de publicaciones del plantel estudiantil según revistas, la Revista Científica Ciencia Médica es la que contabiliza mayor presencia de estudiantes con 126 publicaciones (75,00\%), seguida de la Gaceta Médica Boliviana con 34 publicaciones $(20,24 \%$ y con menos artículos con participación de estudiantes la Revista Médica La Paz con 8 publicaciones $(4,76 \%)$, las dos primeras pertenecen a la Facultad de Medicina de la Universidad Mayor de San Simón, lo que podría atribuirse al posible apoyo mutuo, mismo que originaría un efecto positivo en las futuras publicaciones de las siguientes generaciones de egresados.

En el estudio "Producción científica estudiantil en Latinoamérica: un análisis de las revistas médicas de habla hispana indizadas en SciELO, 2011" las universidades con mayor producción científica estudiantil fueron la Universidad Pontificia Católica de Chile, la Universidad Mayor de San Simón (Bolivia), la Universidad de Chile y la Universidad
Peruana Cayetano Heredia ${ }^{12}$, en el caso particular de Bolivia, la Universidad Mayor de San Simón es la institución con mayor participación estudiantil en publicaciones indizadas en SciELO Bolivia, situación que coincide con el presente trabajo.

Otro estudio sobre la "Apreciación de estudiantes de Medicina latinoamericanos sobre la capacitación universitaria en investigación científica" $60,8 \%$ de estudiantes encuestados, consideraron la existencia de limitaciones para publicar desde el pregrado, considerando a la falta de apoyo docente e incentivos académicos las principales causas ${ }^{13}$, de igual forma, otros trabajos reflejaron que la región de Ica, Perú. En el periodo de 1998 a 2010, en un estudio bibliométrico sobre la producción científica biomédica, era fácilmente superada por publicaciones realizadas por estudiantes de medicina de Chile ${ }^{14}$.

Considerando otro estudio en una universidad de colombiana un $98,95 \%$ de estudiantes considera que investigar es importante, 90,0\% ha tenido interés por investigar en algún momento de su carrera y $78,94 \%$ no hace investigación ${ }^{15}$ teniendo en cuenta que existe evidencia que estudiantes de pregrado de medicina tienen un nivel aceptable de conocimientos en cuanto al proceso de investigación en sus instituciones ${ }^{16,17}$.

Si bien es fundamental en las Facultad de Medicina incrementar la calidad clínica de los egresados, existe también la necesidad de incorporar médicos-investigadores ${ }^{18}$, a objeto de sembrar cultura de investigar y consecuentemente desarrollo en nuestro país, considerando que la producción científica en nuestro país es baja ${ }^{19-21}$, se deberá trabajar en este tema arduamente.

Es así, que actualmente se reconoce la relación estrecha (no necesariamente causal) entre el desarrollo económico de una nación y la productividad de sus investigadores ${ }^{22}$, por lo referido el presente trabajo no busca obligar a investigar, publicar y hacer partícipes a estudiantes de pregrado, más al contrario busca despertar el espíritu de todo investigador oculto que aún no es descubierto, aportar al desarrollo de Bolivia, con propósito de mejorar la producción científica del país, valorando la importancia y esfuerzo del fomento a la investigación y publicación desde el pregrado, así como durante la formación de la residencia médica ${ }^{23,24}$.

Finalmente, el presente trabajo solo valoró a las revistas en SciELO Bolivia, se deberá profundizar los estudios en otros índices e incluso incluir revistas fuera de la región, asimismo y considerando la situación actual de la participación científica estudiantil en Bolivia es clave la atención a este grupo, son muchas las sociedades científicas estudiantiles existentes en este país poco e incluso nada apoyadas. En contra parte se deberá fomentar la cultura para investigar y publicar con apoyo de estas, además de originar políticas que vinculen la formación médica ligada íntimamente a la investigación y publicación desde el pregrado.

Fuente de financiamiento: autofinanciado

Conflictos de interés: los autores declaramos que no existe conflicto de intereses. 


\section{Referencias bibliográficas}

1.Clouet-Huerta D. Correa Katherine. Investigación médica en pregrado: ¿qué está sucediendo en Chile?. Rev Med Chile 2014; 142(11): 1488-1490.

2.Pachajoa-Londoño, Harry Mauricio, Publicación de artículos originales desde el pregrado en una revista médica colombiana entre 1994-2004. CIMEL Ciencia e Investigación Médica Estudiantil Latinoamericana. 2006; 11(1), 24-26.

3.Rios-González CM. Escasa publicación científica en estudiantes de medicina de Paraguay. Educ Med. 2016; 17(2):80-81.

4. Huamaní, C. Chávez-Solis, P. Mayta-Tristán P. Aporte estudiantil en la publicación de artículos científicos en revistas médicas indizadas en SciELO-Perú, 1997 - 2005. An Fac med. 2008. 69(1):42-45.

5. Angulo, R, Angulo, F, Huamaní, C, MaytaTristán, P. Publicación Estudiantil en Revistas Médicas Venezolanas, 2001 - 2005. CIMEL Ciencia e Investigación Médica Estudiantil Latinoamericana. 2008; 13(1):6-8.

6. Carvajal Tapia AE. Importancia y reflexiones sobre la investigación y publicación científica desde pregrado. Revista SCientifica, 2014; 12(1): 7-8.

7. Gozalo Delgado M. León Del Barco B. La promoción de la autoeficacia en el docente universitario. Revista Electrónica Interuniversitaria de Formación del Profesorado. 1999; 2(1): 80-90.

8. Soto Arango, DE, Forero Romero, A. La Universidad Latinoamericana y del Caribe en los desafíos del Siglo XXI. Revista Historia de la Educación Latinoamericana. 2016; 18(26): 279309.
9. Mayta-Tristán P. Enseñando a publicar desde el pregrado. Rev. Méd. Risaralda 2013; 19(1):2-3.

10. Carvajal Tapia AE. Investigación y la formación Científica en pregrado de Medicina. Rev. Discov. Med. 2017; 1(1):57-60.

11. Richard, E. y D. I. Contreras Zapata. El rol de la investigación universitaria en la descolonización e independencia académica: Lo que no se publica no existe....UMSA. Revista Tribuna Docente, 2014; 2(1): 3-5.

12. Taype-Rondán A. Palma-Gutiérrez E., PalaciosQuintana M., Carbajal-Castro C., Ponce-Torres C. Producción científica estudiantil en Latinoamérica: un análisis de las revistas médicas de habla hispana indizadas en SciELO, 2011. FEM 2014; 17(3): 171177.

13. Mayta-Tristán P. et al. Apreciación de estudiantes de Medicina latinoamericanos sobre la capacitación universitaria en investigación científica. Rev Med Chile 2013; 141(6): 716-722.

14. Arroyo-Hernández H. Análisis bibliométrico de la producción científica biomédica en la Región de Ica, Perú. 1998-2010. Revista Médica Panacea. 2011; 1(1): 2-8

15. Ángel Isaza, AM, Botero Suárez, HF, Carolina González, D, Piedad Ospina, L, Velasco, MM, Ocampo, MF. Interés de los estudiantes de medicina por la investigación. Ciencia e Investigación Médica Estudiantil Latinoamericana. 2010; 15(1): 9-13

16. K Diaz C, Manrique LM, Galán E, Apolaya M. Conocimientos, actitudes y prácticas en investigación de los estudiantes de pregrado de facultades de medicina del Perú. Acta Med Peru.
2008; 25(1): 9-15.

17. Ramos-Rodriguez MI, Sotomayor R. Realizar o no una tesis: razones de estudiantes de medicina de una universidad pública y factores asociados. Rev. Peru. Med. Exp. Salud Pública. 2008; 25(3): 322-24.

18.- Larrazabal Córdova C. La investigación y el pregrado. Gac Med Bol 2014; 37(1): 5

19. Cañedo Andali R. Cuba, Iberoamérica y la producción científica en salud en la base de datos PubMed en el período 1999-2008. ACIMED. 2009; 20 (1): 1-27.

20. Eróstegui Revilla C. Evaluación de la difusión de la producción científica en Bolivia. Gac Med Bol 2011; 34(1): 5 .

21. Carvajal Tapia AE. Una visión panorámica de la productividad científica en salud de Bolivia. Rev Med La Paz. 2017; 23(2) 88-90.

22. Niaz Mansoor. Investigación y la riqueza de una nación. Revista Interciencia. 2000; 25(1) 3740.

23. Christakis D, Davis R, Rivara F. Pediatric evidence-based medicine: Past, present, and future. J Pediatr. 2000; 136(3): 383-9.

24. Carvajal Tapia AE, Carvajal Rodríguez E. Valoración de la participación científica de pediatría en Latinoamérica, una aproximación con el uso de Scimago Journal \& Country Rank 1996-2016. Arch Argent Pediatr. 2018; 116(2): 356-358. 